\title{
Ultrastructural study of the coccidian Cryptosporidium sp. from stomachs of juvenile cichlid fish
}

\author{
Jan H. Landsberg ${ }^{1}$ \& Ilan Paperna ${ }^{2}$ \\ ${ }^{1}$ Laboratory for Research of Fish Diseases, Nir-David 19150, Israel \\ ${ }^{2}$ Dept. of Animal Sciences, Faculty of Agriculture of the Hebrew University of Jerusalem, Rehovot 76-100, Israel
}

\begin{abstract}
Infection by the coccidian Cryptosporidium sp. was found in the stomach of cultured cichlid fry and fingerlings. Merogony and gamogony stages were located at the microvillous zone of the superficial cells of the stomach epithelium while oogony and sporogony stages were located intracellularly within the mucosal epithelium. Epithelially attached stages were covered by a microvillous membrane. These structural and developmental characteristics have not been reported previously in other Cryptosporidium species
\end{abstract}

\section{INTRODUCTION}

Cryptosporidiosis has been reported frequently in mammals as well as from a number of birds and reptiles (Levine 1984a). Only 2 reports are currently known from fish: that from the tropical marine species Naso lituratus (Hoover et al. 1981) and that from the freshwater carp Cyprinus carpio (Pavlásek 1983). The present communication reports on cryptosporidian organisms of fry and fingerlings of cichlids which sporulate within the stomach mucosa. This type of development has not been reported previously in other Cryptosporidium species.

\section{MATERLALS AND METHODS}

Coccidian infections were detected in the stomachs of hatchery-reared fry and fingerlings of Oreochromis aureus (Steindachner) $\times O$. niloticus (L.) hybrids (Cichlidae). In fish 11 to $55 \mathrm{~mm}$ in length, incidence of infection was high $(58.8 \%, \mathrm{n}=17)$, whereas fish 56 to $152 \mathrm{~mm}$ in length $(\mathrm{n}=10)$ were not infected. Infection was determined by light microscopic examination of unstained squash (Nomarski interference illumination optics) and Giemsa-stained smear preparations of stomach, anterior, mid and post intestine, and faecal casts. Five fish fry and stomach tissue from another 5 fish were fixed in $10 \%$ buffered neutral formalin and embedded in glycol methacrylate (Lulham 1979). Sections 3 to $6 \mu \mathrm{m}$ thick were cut using a Sorval JB-4 glass knife microtome and stained either by Meyer's haemalum-eosin or PAS methods. For transmission electron microscopy (TEM), infected stomachs from 3 fish were fixed in Karnovski's fixative for $24 \mathrm{~h}$ at $4^{\circ} \mathrm{C}$. After intensive rinsing in cacodylate buffer $(0.1 \mathrm{M}, \mathrm{pH}$ 7.4), tissues were post-fixed in $1 \%$ osmium tetroxide in the same buffer. After further rinsing, also in the same buffer, tissues were dehydrated in an ascending series of alcohols and embedded in Epon. Thin sections, cut on an LKB III ultratome with a diamond knife, were stained on the grid with aqueous uranyl acetate and lead citrate and examined with a Jeol TEM 100 CX.

\section{RESULTS AND DISCUSSION}

\section{Light microscopy}

Parasites appeared either as dense spherical or 'mushroom-like' structures attached to the villous border of the stomach mucosal epithelium (identified by TEM as meronts or gamonts), or were located singly or in clumps within the epithelial cells or deep within the epithelial tissue of the stomach (Fig. 1). These latter stages, identified by TEM as zygotes and sporulated oocysts, contained numerous PAS-positive granules.

Meronts and gamonts did not appear to cause notice- 

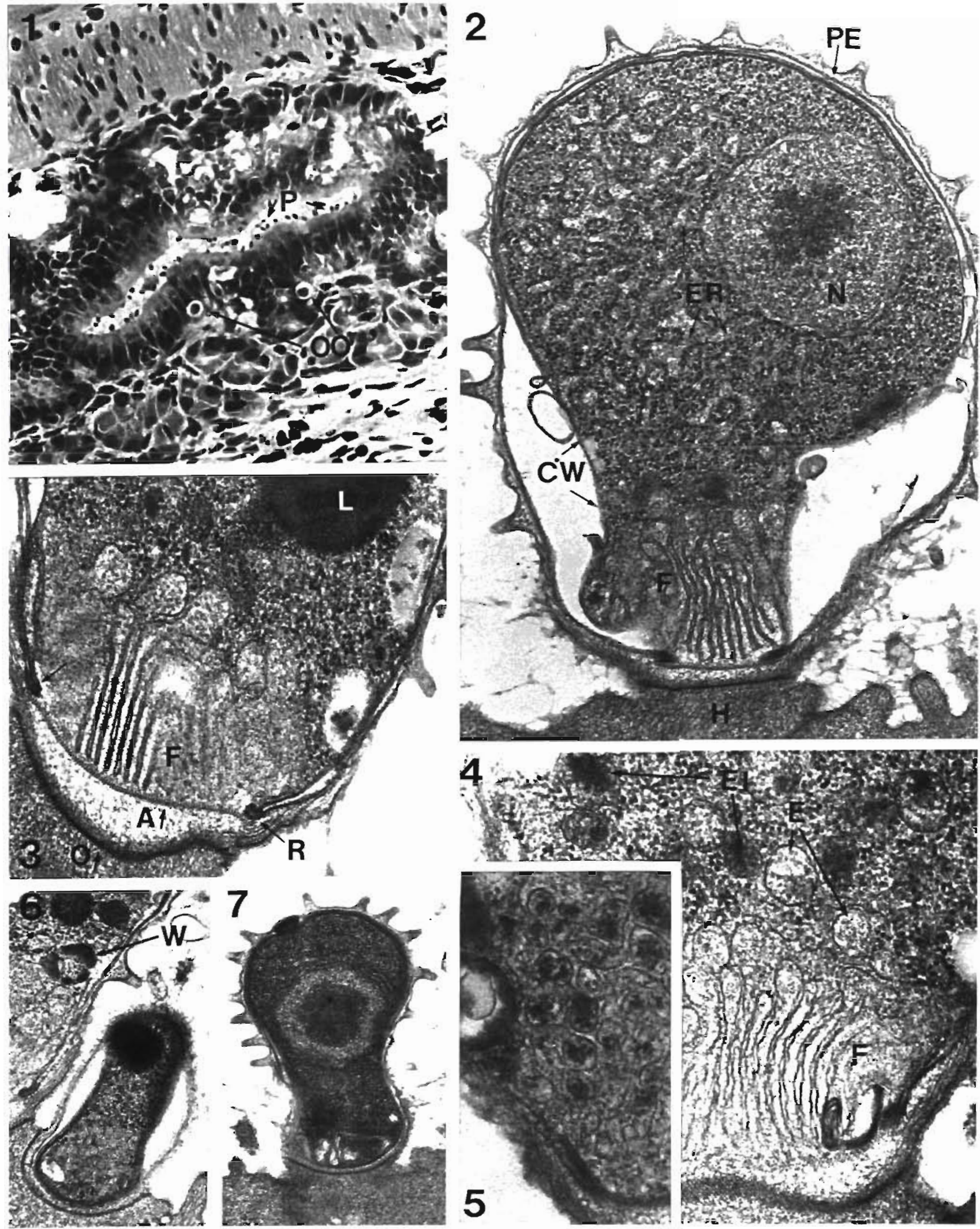

Fig. 1 to 7. Cryptosporidium sp. Fig. 1. Histological section through the stomach of Oreochromis aureus $\times$ O. niloticus naturally infected with Cryptosporidium sp. OO: intracellular oocysts surrounded by necrotic cells; P: epithelial stages. LM, $\times 600$. Fig. 2 Early uninucleate meront. CW: parasite plasmalemma; ER: rough endoplasmic reticulum; F: feeder organelle; $H$ : host cell; $N$ : parasite nucleus; PE: parasitophorous membrane. TEM, $\times 29300$. Fig. 3. Attachment zone of early gamont; A: inner band; F: feeder organelle; L: lipid vacuole; O: outer band; R: plaques. TEM, $\times 36$ 400. Fig. 4. Attachment zone of a meront showing feeder organelle $(F)$. E: empty endocytic vesicle; EI: full endocytic vesicle. TEM, $\times 41000$. Fig. 5 . Full endocytic vesicles at the feeder organelle zone. TEM $\times 38900$. Fig. 6. Early uninucleate meront (right) and section of macrogamont (left) showing small electron-dense granules (W). TEM, $\times 22$ 500. Fig. 7. Advanced stage uninucleate meront. TEM, $\times 17700$ 
able pathological changes in the epithelial cells. Infections by oocysts resulted in the eventual necrosis of the host cell. The oocysts remained enclosed within pockets of necrotic cells within the tissue. In very few instances, however, oocysts were seen within degenerate or necrotic cells located close to the surface of the epithelial layer. Very rarely, sloughing of these cells released oocysts into the stomach lumen. However, oocysts were not normally seen either in the intestinal lumen or faecal contents, even in heavily infected fish.

\section{Electron microscopy}

\section{Microvillous membrane}

Meront and gamont stages were covered by a microvillous membrane. Microvilli of the membrane were spaced at regular intervals and contained a dense, finely granular substance (Fig. 2). Such a distinct microvillous membrane has not been reported in any other Cryptosporidium species. Neither the microvillous membrane covering the parasite nor the microvillar surface of the stomach mucosa contained the filamentous material or glycocalyx found on both cryptosporidia and microvillar surfaces of mammalian host cells (Vetterling et al. 1971, Pohlenz et al. 1978).

\section{Attachment to host cell}

The 2 electron-dense bands of the attachment zone at the host/parasite interface were usually distinct. In detail, they appeared to consist of electron-dense microfibrils interwoven perpendicularly (Fig. 2, 3 \& 4). The inner band appeared to be connected to the 2 plaques of Vetterling et al. (1971), annular rings of Pohlenz et al. (1978), or osmiophilic connection points of Goebel \& Braendler (1982), rather than being continuous with the interlamellar layer of the parasitophorous envelope as previously reported (Vetterling et al. 1971, Pohlenz et al. 1978, Goebel \& Brandler 1982). The plaque appeared to consist of several electron-dense platelets (Fig. $2 \& 3$ ).

\section{Feeder organelle}

The feeder organelle ('attachment zone' of Vetterling et al. 1971) appeared to consist of multiple parallel pellicular folds with vesiculated widenings at their endings (Fig. 2, $3 \& 4$ ). The folds were more numerous and longer than those seen in the feeder organelles of other cryptosporidia (Vetterling et al. 1971, Pohlenz et al. 1978, Goebel \& Braendler 1982). In appearance, the feeder organelle was reminiscent of the arrays of parallel pinocytic vesiculate tubules found fringing the plasmalemma of myxosporean plasmodia (Current \& Janovy 1976, Current 1979). The vesicular invaginations of the feeder organelle were fringed by what appeared to be endocytic vesicles (Fig. 4), some of which contained a coarse, granular substance (Fig. 4 \& 5). Similar endocytic vesicles were reported by Goebel \& Braendler (1982) in Cryptosporidium sp. from mice.

\section{Attached early developmental stages}

Attached early uninucleate meronts $(\overline{\mathrm{x}}=1.05 \times$ $1.95, \mathrm{SD}= \pm 0.78 \times 0.78$, range $=0.5$ to $1.6 \times 1.4$ to $2.5 \mu \mathrm{m}, \mathrm{n}=2$, in sectional plane) were lacking the microvillous membrane surrounding more advanced stages (Fig. 6). The vesicular component of the feeder organelle filled over half of the cytoplasm. Pellicular folds could not be detected (possibly due to the plane of the section) and only vague outlines of the junction bands were traceable. In a more advanced developmental stage $(\overline{\mathrm{x}}=2.57 \times 3.52, \mathrm{SD}= \pm 0.69 \times 0.5$. range $=1.2$ to $3.46 \times 3.0$ to $4.6 \mu \mathrm{m}, \mathrm{n}=9$, in sectional plane) the microvillous membrane covering the parasite was already present and pellicular folds of the feeder organelle were apparent (Fig. 7).

\section{Meronts, merogony and merozoites}

Meronts in all stages of differentiation $(\overline{\mathrm{x}}=2.67 \times$ $3.52, \mathrm{SD}= \pm 0.39 \times 0.24$, range $=2.4$ to $3.1 \times 3.1$ to $3.7 \mu \mathrm{m}, \mathrm{n}=6$, in sectional plane) were similar in their fine structure to meronts described from other cryptoporidia (Vetterling et al. 1971, Proctor \& Kemp 1974, Pohlenz et al. 1978, Bird \& Smith 1980, Current 1984). This applies to: the extensive network of rough endoplasmic reticulum (Fig, 2); the absence of mitochondria; fine structural changes taking place during merogony leading to the formation of either 4 or 8 merozoites (Fig. 8 to 11): and the fine structure of the merozoites $(\overline{\mathrm{x}}=0.76 \times 3.5, \mathrm{SD}= \pm 0.14 \times 0.0$, range $=0.6$ to $0.88 \times 3.5 \mu \mathrm{m}, \mathrm{n}=3$, in sectional plane) (Fig. 11). In advanced meronts prior to cytokinesis, pseudopodium-like processes extended from the pellicle into the parasitophorous vacuole (Fig. 8). Such processes have not been reported previously from other Cryptosporidium species.

A thick, electron-dense subpellicular layer was present in the region of developing merozoites (Fig. 8 \& 9). This layer appeared in the early meront and formed the subpellicular bilaminated membrane in the budding and fully developed merozoites (Fig. 10 to 11). A subpellicular amorphous layer seen in early uninucleate 

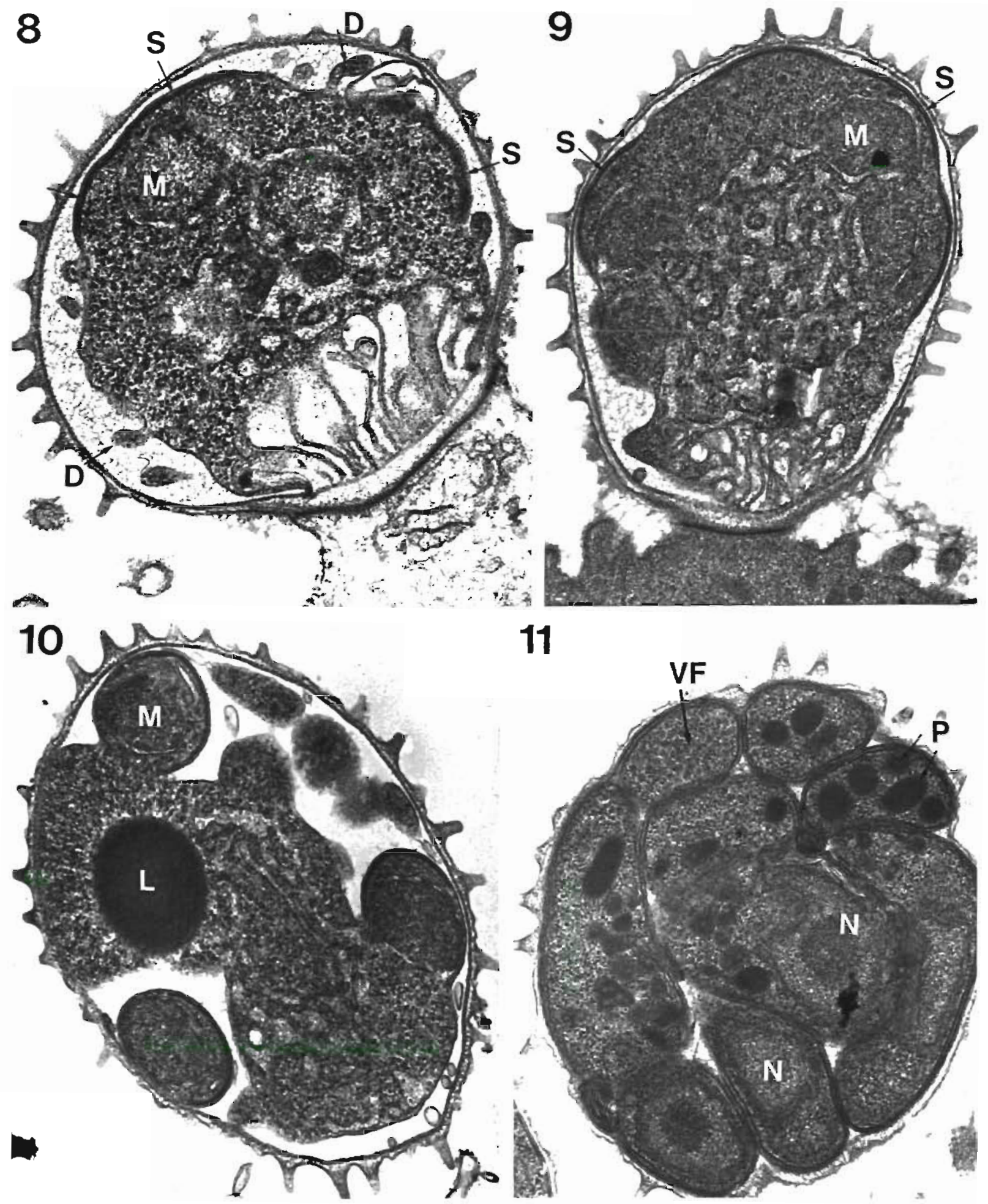

Fig. 8 to 11. Cryptosporidium sp. Fig. 8. Early differentiating meront. D: pseudopodium-like processes; M: differentiating merozoite; S: thick subpellicular layer TEM, $\times 24600$. Fig. 9. Early stage merogony. $M$ : differentiating merozoite; S: thick subpelliculas layer TEM, $\times 22$ 300. Fig. 10. Meront containing 4 merozoites. L: lipid vacuole; M: differentiatıng merozoite. TEM, $\times 24$ 100. Fig. 11. Meront containing 6 to 8 merozoites. N: nucleus; P: rhoptries; VF: presumed primordia of the feeder organelle. 
meronts could be the primordium of the subpellicular layer of the meronts (Fig. 7). Subpellicular layers reported in other cryptosporidia (Vetterling et al. 1971, Bird \& Smith 1980) were not as pronounced as those presently described. Aggregated inclusions at one pole of the merozoite which contained a fine electron-dense substance appeared to be the primordia of the feeder organelle (Fig. 11).

\section{Macrogamonts}

Macrogamonts $\overline{(\bar{x}}=3.07 \times 4.82, \mathrm{SD}= \pm 0.92 \times$ 1.31 , range $=2.2$ to $4.8 \times 3.4$ to $7.0 \mu \mathrm{m}, \mathrm{n}=9$, in sectional plane) (Fig. 12) possessed a nucleus with distinct nucleolus, numerous amylopectin bodies, a large single lipid vacuole (Fig. 3), a large, single membrane-bound inclusion of fine granular content (Fig. 12 \& 14), numerous small round inclusions of electrondense globular material (Fig. 12 \& 13), and duplication of the pellicle or formation of a subpellicular layer (Fig. 14) (Vetterling et al. 1971, Barker \& Carbonell 1974 , Bird \& Smith 1980, Goebel \& Braendler 1982). A mitochondrion such as that seen in other cryptosporidia (Goebel \& Braendler 1982) was not detected. The small electron-dense inclusions also formed lobate structures (Fig. 6). These organelles were called wallforming bodies Type I by Vetterling et al. (1971) and Goebel \& Braendler (1982) or were implicated as functional wall-forming organelles (Bird \& Smith 1980). In our opinion, the single, large, membrane-bound inclusion resembles a wall-forming body Type II since it is enclosed within a cisterna of endoplasmic reticulum. Identification of these organelles as wall-forming bodies is presumptive since the process of oocyst wall formation in cryptosporidia is still unknown.

\section{Microgamonts and microgametes}

Microgamonts $(\overline{\mathrm{x}}=2.95 \times 4.1, \mathrm{SD}= \pm 0.21 \times 0.42$, range $=2.8$ to $3.1 \times 3.8$ to $4.4 \mu \mathrm{m}, \mathrm{n}=2$, in sectional plane) contained a dense network of enlarged (electron-lucent), smooth tubules, apparently endoplasmic reticulum (Fig. 15). Such structures have not been reported previously (Vetterling et al. 1971, Bird \& Smith 1980, Goebel \& Braendler 1982). Another distinguishing feature appears to be the occurrence of vesicles in the zone where the feeder organelle is attached to the base of the parasitophorous vacuole (Fig. 15). In all other features, especially with respect to microgamete differentiation, the present species conforms to other cryptosporidia (Vetterling et al. 1971, Bird \& Smith 1980, Goebel \& Braendler 1982). Up to $8 \mathrm{mi}-$ crogametes were seen in single cross sections. The microgametes were similar to those reported by
Goebel \& Braendler (1982) except that the apical pore, adhesion zone and mitochondrion were not located. A small rectangular structure arranged in parallel on each side of the nucleus (comparable to the 'beehive structure' of Goebel \& Braendler 1982) extended throughout the length of the microgamete (Fig. 16). The nuclear membrane was quite distinct. Microgametes were not seen in association with macrogamonts.

\section{Oocysts and sporozoites}

Young zygotes and fully sporulated oocysts containing 4 sporozoites were found only intracellularly within a parasitophorous vacuole deep within the epithelial cells of the stomach mucosa (Fig. 17 to 19). The zygote, or early stage oocyst $(3.5 \times 5.5 \mu \mathrm{m}, \mathrm{n}=1$, in section plane) contained many amylopectín bodies and a single large lipid vacuole. Fully sporulated oocysts $(\overline{\mathrm{x}}=3.25 \times 4.3, \mathrm{SD}= \pm 0.64 \times 0.46$, range $=$ 2.5 to $4.0 \times 3.5$ to $4.7 \mu \mathrm{m}, \mathrm{n}=6$, in sectional plane) were comprised of a double-layered thick wall (which usually shrank following processing) surrounding 4 sporozoites $(\overline{\mathrm{x}}=0.66 \times 3.9, \mathrm{SD}=+0.23 \times 0.27$, range $=0.3$ to $0.9 \times 3.6$ to $4.1 \mu \mathrm{m}, \mathrm{n}=5$, in sectional plane) as well as a residual body of variable size containing amylopectin bodies (Fig. 18 \& 19). The sporozoite had a thick pellicle; an electron-dense cytoplasm containing alternating aggregates of small and large rounded bodies, presumably the rhoptries (or storage substance granules?) and micronemes surrounded by numerous ribosomes (Fig. 20). Apart from locational differences, the mature oocysts conformed ultrastructurally to epicellularly developing thick walled oocysts of other cryptosporidia (Pohlenz et al. 1978, Current 1984). Large aggregates of cellular debris and necrotic substance were present in the parasitophorous vacuole containing the oocysts. More than one oocyst could be seen in a single infected cell (Fig. 17). In some cases, infected cells underwent gradual degeneration (Fig. 17) and eventually disintegrated leaving aggregates of oocysts lying within intercellular spaces. Free oocysts were not normally seen in the intestinal contents or in the faeces.

\section{Taxonomic affinities}

In fish, 2 genera are currently recognized for coccidian infections which develop on the cell surface: Epieimeria and Cryptosporidium; the major difference between them is in the structure of the oocyst (Dyková \& Lom 1981, Levine 1984b). Recent ultrastructural studies of Epieimeria anguillae (Molnár \& Baska 1986) have pointed out further differences between the 2 

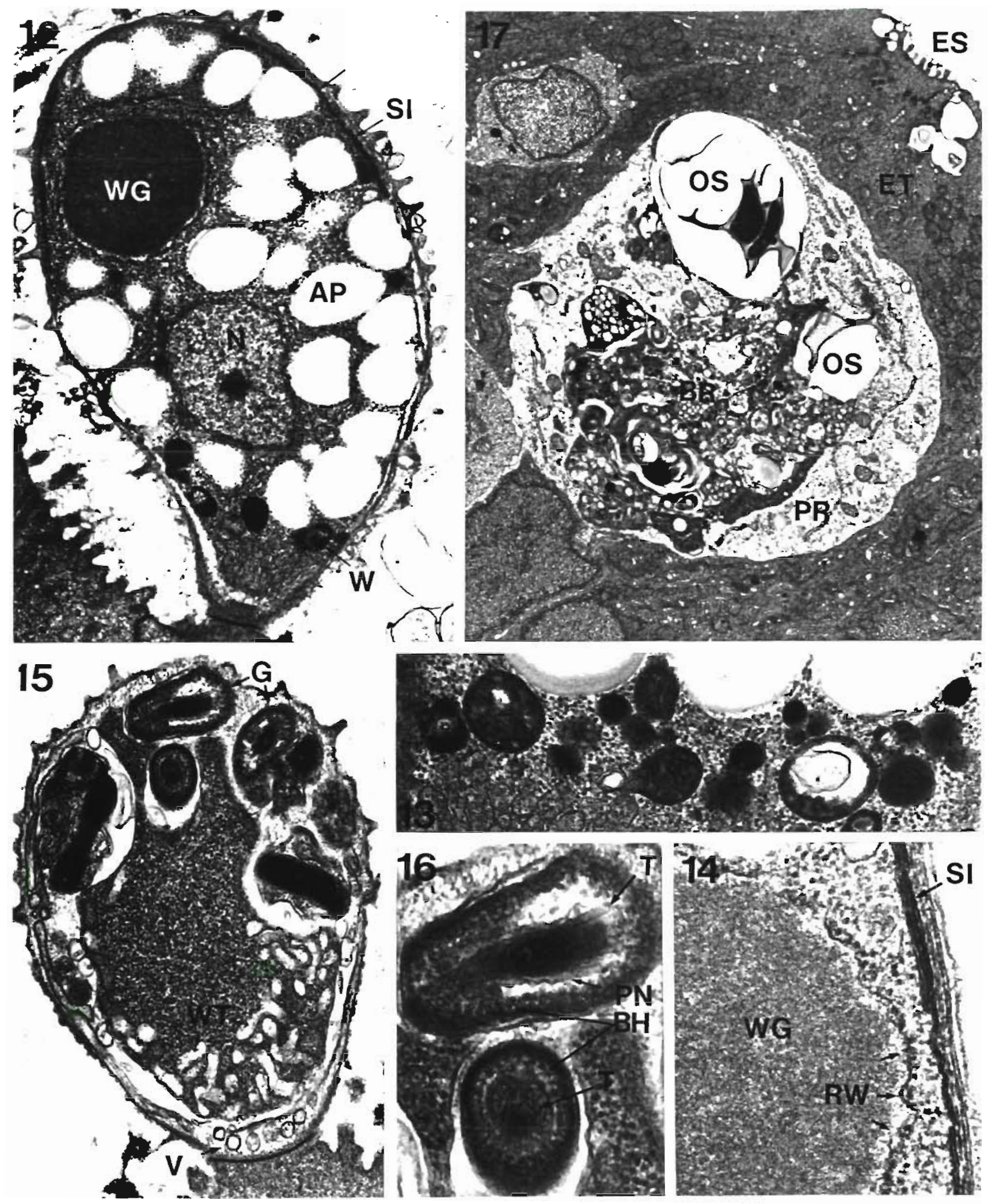

Fig. 12 to 17. Cryptosporidium sp. Fig. 12. Macrogamont. AP: amylopectin granules; N: nucleus; SI: electron-dense subpellicular layer; W: small electron-dense granules; WG: large membrane-bound inclusion. TEM, $\times 15700$. Fig. 13. Small electron-dense inclusions in cytoplasm of macrogamont. TEM, $\times 28900$. Fig. 14. Enlarged view of peripheral zone of membrane-bound inclusion in the macrogamont. RW: cisternal wall fringed by ribosomes; SI: electron-dense subpellicular layer; WG: large membrane-bound inclusion. TEM, $\times 49500$. Fig. 15 . Microgamont. G: microgametes; V: vesicles; WT: smooth endoplasmic reticulum. TEM, $\times 20500$. Fig. 16 . Section through microgametes. BH: 'beehive structure'; PN: nuclear membrane; T: microtubules. TEM, $\times 47$ 100. Fig. 17. Necrotic host cell infected by 2 oocysts. BR: cellular debris and necrotic substance; ES: surface of mucosal epithelium; ET: epithelial cell; OS: oocyst; PR: parasitised host cell. TEM, $\times 5500$ 

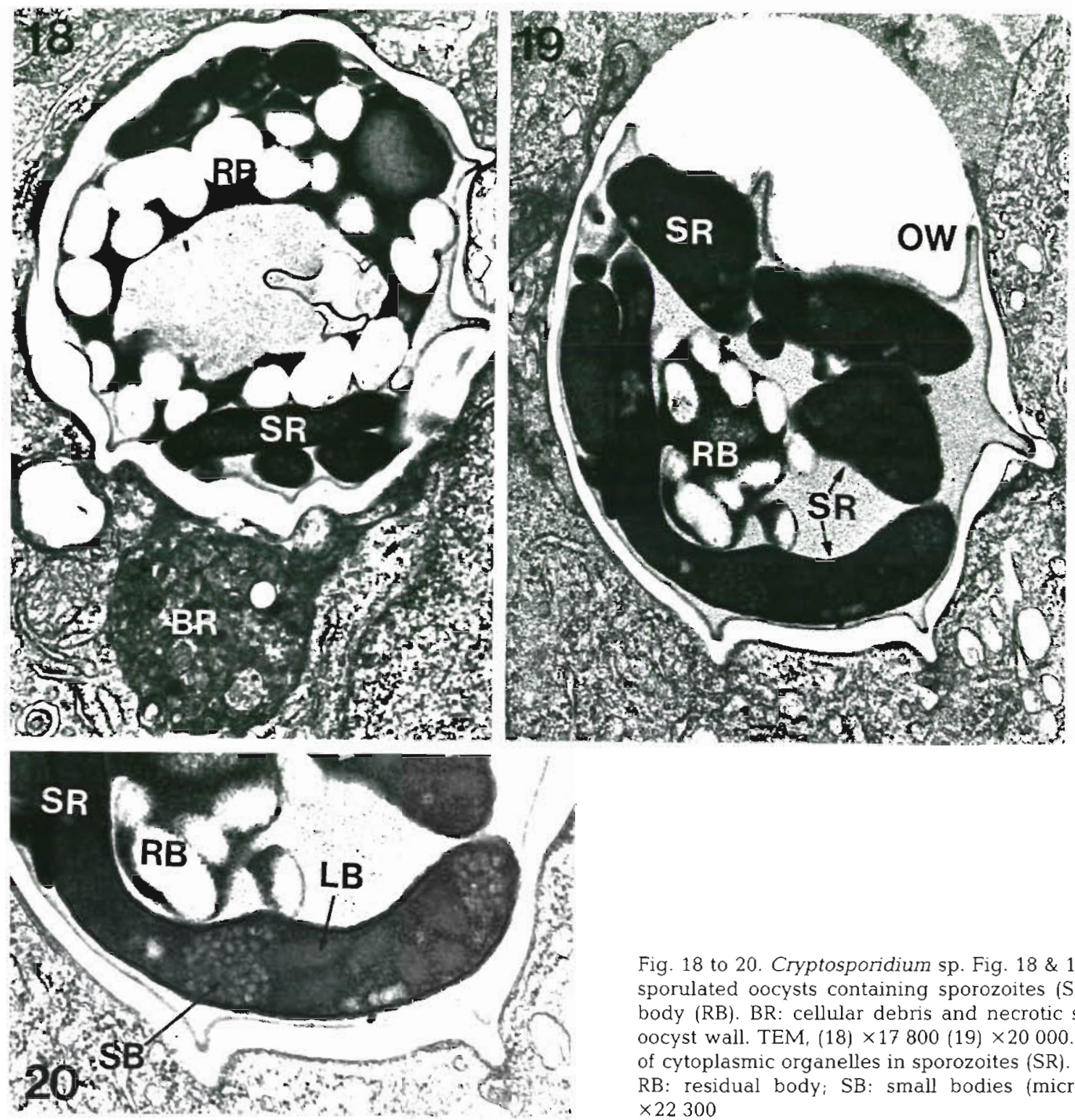

Fig. 18 to 20. Cryptosporidium sp. Fig. 18 \& 19. Thick-walled sporulated oocysts containing sporozoites (SR) and residual body (RB). BR: cellular debris and necrotic substance; OW: oocyst wall. TEM, $(18) \times 17800(19) \times 20000$. Fig. 20 . Details of cytoplasmic organelles in sporozoites (SR). LB: large body; $\mathrm{RB}$ : residual body; $\mathrm{SB}$ : small bodies (micronemes). TEM, $\times 22300$

genera. It should also be mentioned that a type of surface development occurs in Goussia cichlidarum (Paperna et al. 1986) which differs from the above genera in the origin of the membranous layers surrounding the parasite and in the structure of the sporocyst walls.

While the species described here conforms to Cryptosporidium according to the present taxonomic criteria, it differs from all other known species in 2 main respects: (a) oogony and sporogony occur in the tissue; (b) the microvillous membrane covering the parasites and which is apparently derived from remnants of the host cell microvilli is very distinct. This species apparently diverges markedly from the strict structural and developmental conformity exhibited by
Cryptosporidium species from mammals, birds and reptiles (Current 1984, Levine 1984a). Such conformity and the apparent ease with which cross-transmission of cryptosporidia can occur among mammalian or avian host species led Levine (1984a) to reduce all named species into 4 , i.e. one each for mammals, birds, reptiles and fish.

The only other ultrastructurally studied Cryptosporidium sp. from fish is C. nasorum (Hoover et al. 1981). However, fine structural details for this species are insufficient to permit detailed comparisons with the present parasite. The species described here will therefore be referred to as Cryptosporidium $\mathrm{sp}$. until more information on mode of transmission, host specificity and life history becomes available. 
Acknowledgement. Research was supported by a grant from the Volkswagen Foundation through the Ministry of Science and Art of Lower Saxony, Federal Republic of Germany.

\section{LITERATURE CITED}

Barker, I. K., Carbonell, P. I. (1974). Cryptosporidium agni sp. n. from lambs and Cryptosporidium bovis sp. n. from a calf with observations on the oocyst. Z. ParasitenKde 44: 289-298

Bird, R. G., Smith, M. D. (1980). Cryptosporidiosis in man: parasite life cycle and fine structural pathology. J. Pathol. 132: $217-233$

Current, W. L. (1979). Henneguya adiposa Minchew (Myxosporidia) in the channel catfish: ultrastructure of the plasmodium wall and sporogenesis. J. Protozool. 26: 209-217

Current, W. L. (1984). Cryptosporidium and cryptosporidiosis In: Acquired Immune Deficiency Syndrome. Alan R. Liss, New York, p. 355-373

Current, W. L., Janovy, J., JI. (1976). Ulrastructure of the interlamellar Henneguya exilis in the channel catfish. J. Parasit. 62: 975-981

Dyková, I., Lom, J. (1981). Fish coccidia: critical notes on life cycles, classification and pathogenicity. J. Fish Dis. 4: 487-505

Goebel, E., Braendler, U. (1982), Ultrastructure of microgametogenesis, microgametes and gametogamy of Cryptosporidium sp. in the small intestine of mice. Protistologica 18: 331-344

Hoover, H. M., Hoerr, F. J., Carlton, W. W., Hinsman, E. J. Ferguson, H. W. (1981). Enteric cryptosporidiosis in a naso tang, Naso lituratus Bloch and Schneider. J. Fish Dis. 4: $425-428$

Levine, N. D. (1984a). Taxonomy and review of the coccidian genus Cryptosporidium (Protozoa, Apicomplexa). J. Protozool. 31: 94-98

Levine, N. D. (1984b). The genera Cryptosporidium and Epieimeria in the coccidian family Cryptosporidiidae (Protozoa: Apicomplexa). Trans. Am. microsc. Soc. 103: 205-206

Lulham, C. N. (1979). Glycol methacrylate embedding for light microscopy. J. Histotechnol. 2: 68-71

Molnár, K., Baska, F. (1986). Light and electron microscope studies on Epiejmeria anguillae (Léger and Hollande, 1922), a coccidium parasitizing the European eel, Anguilla anguilla L. J. Fish Dis. 9: 99-110

Paperna, I., Landsberg, J. H., Feinstein, N. (1986). Ultrastructure of the macrogamont of Goussia cichlidarum Landsberg and Paperna, 1985, a coccidian parasite in the swimbladder of cichlid fish. Annls Parasit. hum. comp. 61: $511-520$

Pavlásek, I. (1983). Cryptosporidium sp. in Cyprinus carpio Linne, 1758 in Czechoslovakia. Folia Parasitol. (Praha) 30: 248

Pohlenz, J., Bemrick, W. J., Moon, H. W., Cheville, N. F. (1978). Bovine Cryptosporidiosis: a transmission and scanning electron microscopic study of some stages in the life cycle and of the host-parasite relationship. Vet. Pathol. 15: 417-427

Proctor, S. J., Kemp, R. L. (1974). Cryptosporidium anserinum sp. n. (Sporozoa) in a domestic goose Anser anser L., from Iowa. J. Protozool. 21: 664-666

Vetterling, J. M., Takeuchi, A., Madden, P. A. (1971). Ultrastructure of Cryptosporidium wrairi from the guinea pig. J. Protozool. 18: 248-260 